\title{
O PACIENTE DISPNÉICO: sua problemática (PARTE II)
}

\author{
Maria Apparecida Valente*
}

VALENTE, M. A. O paciente dispnéico: sua problemática (parte II). Revv. Esc. Enf. USP, São Paulo, 13(1):89-94, 1979.

A autora apresenta o resultado de estudo sobre o comportamento de pacientes com afeç̧óes pulmonares e cardíacas. ''m fac'c' de crise dispnéica. Faz considerações sobre os problemas de maior incidência e' sobre o comportamc'nto descjável do pessoal de enfermagem.

A enfermeira, desde o primeiro contato com o paciente. além do estabelecimento do «rapport», deve ter sua atenção voltada para uma observação consciente e sistematizada das condições gerais deste. Assistindo pacientes que apresentam queixa de dispnéia, ela deve saber observar os sinais e sintomas mais comuns deste distúrbio. Assim sendo, poderá se dirigir seguramente para a problemática desses pacientes, individualizando-os para o planejamento da assistência de enfermagem.

Neste estudo, a problemática está centrada nas informações de pacientes quanto à dispnéia, relacionadas ao seu estado físico e psicológico. São elas: locomoção. tosse, tempo que têm «falta de ar». período do dia e condições em que se manifesta a dispnéia. posição de maior alívio e posição adotada em casa nas crises de dispnéia, o que costumava fazer em casa nas crises dispnéicas, o que sente e o que pensa quando têm dispnéia, quando foi e como foi a última crise de dispnéia. se solicitou a enfermagem na última crise de dispnéia e os motivos da não solicitação.

A locomoção é importante para a eficiência do cuidado ao paciente com dispnéia. Os pacientes em repouso no leito, tanto relativo como absoluto, podem ter outras afeç̧ões associadas à principal; além disso, outras necessidades básicas poderão estar afetadas, o que contribuiria para o aumento de sua problemática. Verificou-se neste trabalho, que 26 pacientes $(31,3 \%)$ estavam em repouso no leito: todos apresentavam dispnéia em pequenos esforços ou $\mathrm{cm}$ repouso, sendo que oito tinham dispnéia constante.

A tosse, sinal freqüente de doença pulmonar ou cardíaca. foi verificada em $73.5 \%$ dos pacientes; era, na maioria dos casos, produtiva. SECOR ${ }^{21}$ afirma ser a tosse persistente um importante sinal de presença de afecção pulmonar ou cardíaca. A tosse pulmonar geralmente é cansativa e acompanhada de secreção, enquanto a tosse cardíaca é mais leve. Além disso, a tosse produtiva, com expectoração de escarro. ocorre em $90 \%$ dos pacientes que apresentam tosse persistente: já a tosse não associada à hipersecreção ocorre. em geral. nas doenças cardíacas ${ }^{15}$.

Cabe à equipe de enfermagem estimular e ajudar o paciente com tosse produtiva a tossir para expelir a secreção: fornecer material necessário à sua higiene brônquica, orientando-a quanto aos princípios higiênicos para o seu conforto e o

\footnotetext{
* Professor Assistente da disciplina Enfermagem Médico-Cirúrgica da EEUSP. Mestre em Enfermagem.
} 
dos companheiros da enfermaria; controlar a quantidade de secreção para poder observar a evolução da doença e participar de sua terapêutica. Quando a tosse for seca, podendo intensificar a dispnéia, deve procurar acalmar o doente, medicando-o conforme a prescrição médica.

A extensão do tempo em que o paciente vem apresentando dispnéia é um dado importante para a equipe de enfermagem, pois, como já foi mencionado anteriormente, o paciente, longe de se acostumar à dispnéia, torna-se cada vez mais assustado à sua aproximação. Quanto a este tópico, verificou-se, no estudo feito, que o tempo variou de menos de um ano, abrangendo um mínimo de 8 dias, a mais de dez anos, alcançando até 30 anos (esta última informação prestada por três pacientes). Casos de dispnéia recente parecem estar ligados aos pacientes com afecções agudas ou àqueles que estão entrando na fase de descompensação respiratória.

Pacientes com doença pulmonar obstrutiva crônica, conforme comenta BETHLEM ${ }^{4}$, começan $\_$a se descompensar, apresentando dispnéia quando cerca de $50 \%$ do parênquima pulmonar está alterado, evoluindo progressivamente.

Ertre os pacientes que há mais tempo se queixam de falta de ar, encontramse os mais idosos, no meio dos quais figuram pacientes com afecção pulmonar e comprometimento cardíaco direito (cor pulmonale), e outros com insuficiência cardíaca associada à complicação pulmonar (edema pulmonar). Segundo SILBER \& KATZ ${ }^{22}$, é difícil determinar a origem da dispnéia em pacientes idosos, porque tanto os pulmões como o coração geralmente estão comprometidos.

Convém notar que há uma correlação entre a extensão do tempo em que o indivíduo vem se queixando de falta de ar e a ansiedade por ele expressada, ambas aumentando paralelamente. Este dado, de relevante importância, não deve passar desapercebido da enfermeira para a programação da assistência do paciente.

$O$ período do dia em que ocorre a falta de ar, a sua relação com o esforço e a posição preferencial adotada pelos pacientes são dados que devem ser considerados pela equipe de enfermagem para um melhor atendimento.

A maioria dos pacientes consultados informou que a dispnéia surge a qualquer hora, manifestando-se nos pequenos esforços ou mesmo em repouso; esses pacientes geralmente se mantêm no leito, em posição mais elevada. Também foi alta a incidência de informações de dispnéia durante a noite, estando muito associada ao repouso.

A dispnéia em repouso, em geral, é produzida por atelectasia, asma, pneumotórax, enquanto a dispnéia de esforço pode acompanhar qualquer condição que restrinja a ventilação. A dispnéia paroxística noturna costuma acordar o paciente, deixando-o em estado angustioso, com os olhos arregalados, sudorese fria, tosse seca ou com escassa expectoração viscosa; obriga-o a sentar-se no leito e a tomar respirações profundas, ou a levantar-se à procura de ar, numa janela aberta. É, geralmente, causada por insuficiência ventricular esquerda, por estenose mitral ou insuficiência aórtica, mas pode também ocorrer em pacientes com processo obstruti. vo crônico (asmáticos, bronquíticos e enfisematosos) $1,4,5,6.7,11,13,17,18,19,20,21,23,24$.

Quando em casa, com mais liberdade de ação, o individuo por certo procura posição mais confortável nos seus momentos difíceis. Sendo assim, procurou-se constatar a coincidência das resposta quanto à posição adotada em casa, em crise dispnéica, e quanto à posição declarada de alívio, pelo paciente. Houve $81,9 \%$ de 
respostas coincidentes, incidindo $69.9 \%$ delas na posição «sentada ou semi-sentada». A enfermeira, ao planejar os cuidados de enfermagem. deve considerar as preferências dos pacientes quanto à posição. pois assim estará atendendo à necessidade de oxigenação e proporcionando o conforto necessário ao doente.

Considerando-se que a dispnéia é uma situação angustiante e que por isso exige dos pacientes, nesses momentos, as medidas para saná-la. investigou-se o que eles costumavam fazer em casa nessas ocasióes. A maioria dos pacientes, 46 $(55,4 \%)$. fazia uso de medicamentos. Dentre estes, mais da metade não sabia informar qual o medicamento que tomava - destes, 14 (16,9\%) disseram possuir receita médica e $10(12.0 \%)$, serem medicamentos indicados na farmácia, Os que sabiam informar o nome do medicamento disseram tomar broncodilatadores, digitálicos, vasodilatadores, diuréticos e analgésicos, medicamentos estes que podem ocasionar variados efeitos colaterais. necessitando. portanto, de controle médico ou de enfermagem.

A falta de orientação desses pacientes é fato importante, devendo a equipe de saúde estar ciente para melhor esclarecê-los quanto aos perigos da auto-medicação indiscriminada. Como esses pacientes geralmente permanecem hospitalizados por longo tempo, sugere-se que enquanto internados, ele e seus familiares sejam orientados quanto à medicação e aos cuidados a serem observados, assim como quanto a outras medidas de alívio e conforto a serem adotadas em casa. Dois pacientes informaram ter torpedo de oxigênio em casa. Como a oxigenoterapia pode ser feita em casa, cabe à enfermeira orientar os pacientes e seus familiares no que diz respeito aos efeitos paradoxais desta terapêutica. tendo em vista sobretudo a prevenção de uma possível dependência. Dez pacientes $(12.1 \%)$ tomavam chás caseiros variados; quanto aos pacientes que informaram nada tomar $\left(30,1^{\%} \%\right)$. a justificativa era de que a dispnéia cessava com o repouso.

Saber o que o paciente sente nos momentos em que se intensifica a falta de ar pode ajudar o enfermeiro a compreendê-lo e assistí-lo. Foi interessante observar que as informações. neste tópico, foram variadas, mas que o grupo mais numeroso referiu-se a «canseira», sufocação, nervosismo e ansiedade, «batedeira». dor no peito, «falta de ar»e fraqueza.

Parece que o estado psíquico, além do comprometimento físico desses pacientes, poderia ser fator associado para produzir esses sintomas junto à falta de ar ${ }^{2}$. «Ninguém põe em dúvida que a ansiedade, o medo e outras condições psicológicas contribuem para intensificar a dispnéia» RATTO ${ }^{20}$.

A fadiga muscular expressada pelo paciente como «canseira» acompanha com muita freqüênia a dispnéia e pode ser determinada por vários fatores. Pode ser conseqüência do aumento do esforço para ventilar, de maior consumo de oxigênio pelos músculos respiratórios ou da incapacidade de aumentar o débito cardiaco em face de uma sobrecarga, nos casos de insuficiênica cardíaca congestiva. Nas anemias, o oxigênio transportado pode ser insuficiente para atender às necessidades. determinando a exaustão ${ }^{3.14,20}$.

A taquicardia, mencionada pelo paciente como «batedeira». pode ser explicada como um mecanismo de compensação cardiaca, na tentativa de uma melhor oxigenação sangüinea ${ }^{9}$.

A sensação de sufocação, acompanhada, às vezes, de tontura, nervosismo, aflição ou cefaléia parece estar associada à baixa oxigenação e à estimulação direta do centro respiratório pelo aumento do dióxido de carbono ${ }^{14.24}$. 
Dor no peito, desespero, angústia e muitas outras informações subjetivas relacionadas à dispnéia que o paciente tem apresentado são, na maioria das vezes. difíceis de serem explicadas, embora se justifique sua inquitação. Segundo CROFTON \& DOUGLAS ${ }^{8}$, o paciente com dispnéia está ciente desse distúrbio, porém a forma de reagir ao mesmo é individual.

Segundo FRITTZ JUNIOR ${ }^{12}$, embora o mecanismo de desconforto respiratório seja, em parte, desconhecido, um grande número de doenças associadas à falta de ar são bem caracterizadas, podendo resultar da interação de mecanismos diversos ou do efeito de um único mecanismo.

$O$ pensamento humano trabalha incessantemente $e$, nos momentos mais dificeis, ele se focaliza mais no íntimo dos individuos. À pergunta «no que pensa quando tem falta de ar», foram obtidas variadas respostas que, para efeito da apresentação foram grupadas em cinco: na morte, em Deus e nos familiares; em nada; na doença e tratamento; em melhorar; no trabalho que dou.

Considerando-se a possibilidade de o homem e a mulher reagirem diferentemente em situação de estresse, como é o caso da dispnéia, procurou-se investigar esta questão. Estatisticamente, não houve diferença significativa nas respostas apresentadas por homens e mulheres.

Observou-se que a maioria dos pacientes pensa na morte ,em Deus e nos familiares; dos pacientes que assim pensam, as mulheres constituem a maior parte. Isto parece sugerir que o sentimento da maternidade, sempre exaltado na mulher, faça, nesta hora angustiante, com que ela pressinta a morte e se dirija a Deus, não se desligando de seus familiares. Um grupo de homens parece que assim também reagia; outro, porém, declarou nada pensar, ou pensar na doença e no tratamento ou simplesmente em melhorar. Uma paciente disse que pensava estar «dando muito trabalho».

Vale acentuar que estas respostas foram dadas a uma pessoa a quem eles acabavam de conhecer, embora se tenha procurado estabelecer o melhor «rapport». A enfermeira da clínica, porém, no seu convívio diário com o paciente, terá melhores condições para identificar as reais necessidades psico-físico-espirituais dos pacientes.

Considerando-se o paciente dispnéico como um indivíduo com desconforto intenso associado ao sentimento de pânico, ansiedade, medo de sufocação e morte, a enfermeira deve estar atenta à sua individualização por meio da identificação de seus problemas, a fim de assistí-lo integralmente nas áreas psico-biológica, psicosocial e psico espiritual. São os incidentes desagradáveis, como, no caso, a falta de ar, que exigem da equipe de enfermagem um relacionamento excepcional e intensificado. É nessa ocasião que o paciente recebe a «doação da própria enfermeira», isto é, sua presença familiar pronta para ouvir, dar e receber, e retribui com sua confiança e confidência ${ }^{10}$.

A investigação quanto à última crise de dispnéia e sua intensidade teve como finalidade obter informações mais recentes e provavelmente mais exatas.

Quanto à intensidade, nada pode ser afirmado com precisão. É verdade que toi estabelecida uma hierarquia, baseada nas informações dos pacientes, que a classificava em forte, regular ou fraca. No entanto, as respostas são fundalmentalmente subjetivas, não permitindo, portanto ,uma avaliação exata. Segundo RATTO ${ }^{20}$, assim como há pacientes que têm pouca dor e sofrem muito e outros que têm muita dor e sofrem pouco, também há alguns doentes, com difi- 
culdades respiratórias minimas, que acusam forte dispnéia, enquanto outros, com grandes dificuldades respiratórias, informam ter crise fraca. Para esclarecimento desse fato, seriam necessários estudos mais profundos da gênese e da fisiopatologia da dispnéia, estudos estes que ainda hoje são precários.

Quanto à solicitação da equipe de enfermagem pelos pacientes, quando da última crise dispnéica, constatou-se que $66,3 \%$ a solicitaram e $33,7 \%$ não recorreram a ela. As justificativas quanto à não solicitação foram variadas, sendo que $71,4 \%$ informaram que a dispnéia era fraca e passava sozinha com o repouso, ou, ainda, que não desejavam perturbar os membros da equipe de enfermagem. Parece, no entanto, que esse fato pode estar associado aos pacientes de nivel sócio-econômico mais baixo, o que pode ser confirmado pelos níveis de ocupação - escolaridade, estudados inicialmente. Uma paciente disse que ficou «com medo de repreensão», porque havia desobedecido à orientação recebida. Talvez esta paciente pensasse que não deveria dar trabalho ao pessoal da enfermagem ou talvez não estivesse muito consciente do seu estado de saúde, o que sugere necessidade de reforço na orientação.

Três pacientes declararam não chamar alguém da equipe de enfermagem porque sabiam ligar sozinhos o oxigênio. A equipe de enfermagem precisa estar alerta a este pormenor, orientando muito bem os pacientes quanto ao uso adequado da oxigenoterapia e, sem assustá-los, falar dos perigos decorrentes de seu uso indevido. Além disso, deve observar periodicamente os pacientes e o volume do oxigênio que estão recebendo.

Embora os pacientes muitas vezes deixem de solicitar a equipe de enfermagem, sabe-se que, quando entram em crise dispnéica, sentem-se angustiados, nervasos, com medo de morrer, sendo, portanto, estas as ocasiões em que a presença de enfermeira se faz mais necessária. Talvez a equipe de enfermagem não deva esperar ser chamada, mas sim estar atenta para compreender e identificar a falta de ar do paciente, dispensando-lhe cuidados, mesmo sem solicitação. Isso faz parte do relacionamento enfermeira-paciente, que tem como objetivo a promoção do conforto e bem-estar do paciente, o seu melhor entendimento e percepção de sua atual condição ${ }^{16}$.

$\mathrm{Na}$ parte III serão abordadas as expectativas especificas do paciente dispnéico quanto à assistência de enfermagem.

VALENTE, M. A. Dispneic patient: their's problematic (part II). Rev. Esc. Enf. USP, São Paulo, 13(1):89-94, 1979.

The author presents the results of a study of the reactions of cardiac and pulmonary patients to an attach of dispnea. She discusses the prevalent problems and the desirable attitude of nursing personnel.

\section{REFERENGIAS BIBLIOGRAFICAS}

1. ASWORTH, P. M. \& ROSE, H. - Cardiovascular disorders: patient care. London, Ballière Tindall, 1973. p. 22-9.

2. BELAND, I. L. - Clinical nursing: pathophysiological and psychosocial approaches. London, MacMillan, 1969. p. 455.

3. BERTOLASI, C. A. \& TRONGE, J. E. - Unidad coronaria: rol dela enfermera. Buenos Aires, InterMédica Editorial, 1972. p. 37-107.

. BETHLeM, N. - Pneumologia. São Paulo, Atheneu, 1973. p. 116, 335

5. BEVILACQUA, F. et alii - Manual de fisiopatologla clínica. Rio de Janeiro, Atheneu, 1974. p. 196 $201,307$.

6. CARRAL, R. T. - Semiologia cardiovascular. 5. ed. México, Interamericana, 1968. p. 3-27. 
7. CECIL, R. L. \& LOEB, H. S. - Tratado de medicina interna. 12. ed. México, Interamericana, 1968. p. 586 .

8. CROFTON, J. \& DOUGLAS, A. - Enfermedades respiratórias. Barcelona, Marin, 1971. p. 46-60.

9. FARRERAS VALENTI, P. - Medicina interna: compendio práctico de patologia medica y terapeutica clinica. 8 cd. Barcelona, Miarin, 1972. v. 1, p. 332-7, 637.8.

10. FERLIC. A. - Existencial approach in nursing. Nurs. Outlook, New York, 16 (10): 30-3, Oct. 1968. (HORTA W. de A. trad. Abordagem existencialista em enfermagem. Enf. Novas Dimens., 2 (3): 175-81, jul./ago., 1976).

11. FOWKES, W. C. \& HUNN, W. K. - Clinical assessment for the nurse practioner. Saint Louis, Mosby, 1973. p. 18-9.

12. FRITTZ JUNIOR, $\mathrm{H}$. W. - Alteraçôes funcionais da respiração e da circulacão: dispnéia. In: HARRISON'S Medicina interna. 6. ed. Rio de Janeiro, Guanabara Koogan, 1974. v. 1. p. 213-6.

13. GANONG, W. F. - Fisiologia médica. 2. ed. São Paulo, Atheneu, 1972 . p. 509-15, 545.

14. HANCHETT, E. S. et alii - Early signs of congestive heart failure. Amer. J. Nurs., New York, 68: $1456-61$, July, 1968 .

15. HARRISON, T. R. et alii - Alterações funcionais da respiração e da circulação: cianose, hipóxia e policitemia. In: HARRISON'S Medicina interna. Rio de Janeiro, Guanabara Koogan, 1974. v. 1 , p. 217-24.

16. JOURARD, S. M. - How well do you know your patient? Amer. J. Nurs., New York, 59: 1568-71, Nov., 1959.

17. MACBRYDE, C. M. \& BLACKLOW, R. S. - Sinais e sintomas: fisiopatologia aplicada e interpretação clínica. 5. ed. Rio de Janeiro, Guanabara Koogan, 1975. p. 327-43.

18. MELTZER, L. E. et alii - Cuidados intensivos para el paciente coronario. México, La Prensa Médica Mexicana, 1973. p. 85.

19. MODELL, W. et alii - Manual de cardiologia para enfermeiras. 5. ed. México, La Prensa Médica Mexicana, 1969. p. 48-9, 177.

20. RATTO, O. R. - Pneumologia. In: MARCONDES, M. et alii - Clínica médica: propedêutica e fisiopatologia. Rio de Janeiro, Guanabara Koogan, 1976 p. 185-266.

21. SECOR, J. - Patient care in respiratory problems. Philadelphia, Saunders, 1969. p. 43-5.

22. SILBER, E. N. \& KATZ, L. N. - Heart disease. New York, MacMillan, 1975, p. 451-2.

23. VEREL, D. - Essential cardiology. Great Britain, Medical \& Technical Publishing, 1973. p. 27, 80

24. WOOD, P. - Enfermedades del corazön y de la circulación. Barcelona, Toray, 1971. p. 326-8. 